\title{
Acompañamiento y empoderamiento: factores clave para el desarrollo de iniciativas empresariales en la comunidad de los JAtNEI DtONA
}

DOI: https://doi.org/10.21158/01208160.n88.2020.2500

\author{
Omar Alonso Patiño C. \\ Universidad Ean \\ opatino@universidadean.edu.co \\ Catalina Lucía Ruíz-Arias \\ Universidad Ean \\ clruiz@universidadean.edu.co \\ Paula Echeverry-Pérez \\ Universidad Ean
}

Fecha de recepción: 23 de septiembre de 2019 Fecha de aprobación: 27 de abril de 2020

Cómo citar este artículo / To reference this article / Comment citer cet article / Para citar este artigo:

Patiño, O. A.; Ruíz-Arias, C. L.; Echeverry-Pérez, P. (2020). Acompañamiento y empoderamiento: factores clave para el desarrollo de iniciativas empresariales en la comunidad de los JAtNEI DtONA. Revista Escuela de Administración de Negocios, (88), 103-122. DOI: https://doi.org/10.21158/01208160.n88.2020.2500

\section{Resumen}

El presente artículo expone la experiencia en la implementación de conceptos y metodologías de innovación social en el desarrollo de ideas de negocio e iniciativas empresariales sostenibles en organizaciones no convencionales. En este caso específico, una comunidad indígena. Si bien la generación de ingresos no es uno de los ejes fundamentales sobre los cuales se guían las actividades de este tipo de organizaciones, con la incorporación de metodologías de diseño e innovación centradas en el hombre se logra que los individuos entiendan los beneficios personales y colectivos que se desprenden del desarrollo de una iniciativa empresarial y tengan una participación más activa en las distintas actividades conducentes a su puesta en marcha. Se realizó un proceso de acompañamiento, a partir de la metodología del doble hexágono, en el que, mediante visitas de campo y la participación activa de los integrantes de la comunidad, se trabajó a fin de lograr procesos de autoorganización y empoderamiento. Con este documento se demuestra que los principios asociados a la innovación social, así como sus metodologías, brindan la posibilidad de acompañar iniciativas empresariales que respeten las condiciones particulares de la comunidad, de modo que es este el elemento que permite incorporar tales iniciativas como parte de su cultura, lo que genera cambios en los hábitos de las personas y facilita así su implementación exitosa.

Palabras clave: empoderamiento; innovación social; emprendimiento; emprendimiento social; incubación empresarial.

\footnotetext{
${ }^{1}$ Doctor en Ciencias Empresariales con Grado Sobresaliente Cum Laude de la Universidad Nebrija, Magíster en Gestión de Organizaciones de la Universidad de Quebec a Chicoutimí, Especialista en Gestión de la Calidad, la Tecnología y la Producción del Centro de Estudios de Postgrado en Administración de Empresas de la Universidad Politécnica de Madrid, Administrador de Empresas de la Universidad Javeriana. ORCID: http://orcid.org/0000-0002-4897-4897

${ }^{2}$ Diseñadora Industrial de la Universidad Nacional de Colombia, con Maestría en Diseño Estratégico en la Universidad Tecnológica de Delft en Holanda (TU Delft). ORCID: https://orcid.org/0000-0001-9171-9499

${ }^{3}$ Diseñadora Industrial - Universidad de los Andes, Colombia. Master in Innovation Management - Central Saint Martins College - University of the arts London. Certificate in Social Innovation - Portland State University. ORCID: https://orcid.org/0000-0002-9509-864X
} 


\title{
Accompaniment and empowerment: key factors for the development of business initiatives in the JAtNEI DtONA community
}

\begin{abstract}
This article describes the experience when implementing social innovation concepts and methodologies in the development of business ideas and sustainable business initiatives in non-conventional organizations, in this specific case, an indigenous community. Although generating income is not one of the fundamental axes on which the activities of this type of organizations are based, incorporating design and innovation methodologies that are focused on human kind help individuals understand the personal and collective benefits that arise from the development of an entrepreneurial initiative, and motivate them to have a more active participation in the various activities leading to its implementation. Based on the double hexagon methodology, we carried out a process of accompaniment in which the work to achieve processes of selforganization and empowerment were performed by means of field visits and the active participation of community members. This document demonstrates that the principles that are associated with social innovation, as well as its methodologies, offer the possibility of accompanying business initiatives that respect the particular conditions of the community, so that this is the element that allows such initiatives to be incorporated as part of their culture, generating changes in people's habits, thus facilitating a successful implementation
\end{abstract}

Keywords: empowerment; social innovation; entrepreneurship; social entrepreneurship; business incubation.

\section{Acompanhamento e empoderamento: fatores-chave para o desenvolvimento de iniciativas de negócios na comunidade JAtNEI DtONA}

\section{Resumo}

Este artigo apresenta a experiência na implementação de conceitos e metodologias de inovação social no desenvolvimento de idéias de negócios e iniciativas empresariais sustentáveis em organizações não convencionais. Nesse caso específico, uma comunidade indígena. Embora a geração de renda não seja um dos eixos fundamentais sobre os quais se orientam as atividades desse tipo de organização, com a incorporação de metodologias de design e inovação focadas no homem, os indivíduos são capazes de entender os benefícios pessoais e coletivos que são derivados do desenvolvimento de uma iniciativa de negócios e têm uma participação mais ativa nas diferentes atividades que levam ao seu início. Foi realizado um processo de acompanhamento, baseado na metodologia do duplo hexágono, na qual, por meio de visitas de campo e da participação ativa dos membros da comunidade, foi realizado um trabalho para alcançar processos de auto-organização e empoderamento. Este documento demonstra que os princípios associados à inovação social, bem como suas metodologias, oferecem a possibilidade de acompanhar iniciativas de negócios que respeitem as condições particulares da comunidade, de modo que este é o elemento que permite incorporar tais iniciativas como parte do sua cultura, que gera mudanças nos hábitos das pessoas e, assim, facilita sua implementação bem-sucedida.

Palavras-chave: empoderamento; inovação social; empreendedorismo; empreendedorismo Social; incubação de negocios. 


\section{Accompagnement et autonomisation: facteurs clés pour le développement d'initiatives entrepreneuriales dans la communauté JAtNEI DtONA}

\section{Résumé}

Cet article présente l'expérimentation de la mise en œuvre de concepts et de méthodologies d'innovation sociale dédiées au développement d'initiatives entrepreneuriales et durables dans des organisations non conventionnelles. Cette investigation se base sur une communauté indigène pour qui la génération de revenus n'est pas un des pilliers fondamentaux guidant ce type d'organisation humaines, mais qui grâce à l'incorporation de méthodologies de conception et d'innovation centrées sur l'humain, permet aux individus de comprendre les bénéfices personnels et collectifs découlant du développement d'une initiative entrepreneuriale de ce type et les rend plus ouverts aux différentes activités menant au démarrage d'un tel projet. Un processus d'accompagnement à été mis en place se basant sur la méthodologie du double hexagone où, au travers de visites sur le terrain et de la participation active des membres de la communauté, un travail de réalisation des process d'auto-organisation et d'autonomisation a pu être mené. Ce document démontre que les principes associés à l'innovation sociale et leurs méthodologies rendent possible l'accompagnement d'initiatives entrepreneuriales respectant les conditions culturelles particulières des communautés pouvant générer des changements dans les habitudes des individus et faciliter le succès d'initiatives entrepreneuriales.

Mots-clés: autonomisation; innovation sociale; entrepreneuriat; entrepreneuriat social; incubation d'entreprises. 


\section{Introducción}

F $\mathrm{n}$ Colombia habitan cerca de 1400000 indígenas. CDe estos, tan solo 940000 se encuentran en resguardos debidamente protegidos (Organización Nacional Indígena de Colombia [ONIC], 2018; Departamento Administrativo Nacional de Estadística [DANE], 2005). Los restantes no lo están porque han sido víctimas del desplazamiento forzado que ha producido la violencia vivida por el país durante más de 50 años o por actividades esclavizantes, como, por ejemplo, las ejercidas por la industria del caucho, que los han disgregado a lo largo del territorio nacional. Esta situación ha traído como consecuencia que muchas de las comunidades indígenas existentes hace 50 años hayan desaparecido o se encuentren en camino de hacerlo.

En medio de este contexto existen comunidades que buscan reagruparse, algunas de ellas con apoyo del Estado mediante la asignación de tierras y el suministro de condiciones básicas de subsistencia en sus nuevos asentamientos. En esta nueva situación, con los apoyos que brinda el Estado y los recursos provenientes de otros organismos, nacionales e internacionales, las comunidades indígenas beneficiadas han logrado desarrollar procesos que les permiten su sostenimiento y el rescate de las costumbres y los valores propios de la comunidad. La intención del Estado es proteger estas culturas ancestrales, sin embargo, su acción no es suficiente, pues más de la mitad de los indígenas en Colombia están en condición de pobreza y riesgo de desaparecer, por lo cual las comunidades deben establecer procesos que les permitan mejorar su calidad de vida, no dependientes de ayudas externas ni de los recursos que se generan por cuenta de actividades ilícitas tales como el cultivo de coca, el insumo principal de las acciones del narcotráfico.

En este sentido, la formulación y la ejecución de proyectos productivos - no asistencialistas - que permitan el desarrollo de actividades generadoras de recursos para las comunidades, de manera colectiva, y para sus integrantes, es una forma de brindar alternativas económicas diferentes y estables dirigidas a esta parte de la población.

Así las cosas, este proyecto de investigación, realizado en el 2018 en La Fragüita ${ }^{4}$, con la participación activa de los JAtNEI DtONA, tuvo como objetivo general el desarrollo de iniciativas empresariales a partir del diseño de modelos de negocio sostenibles, ajustados a las condiciones, las costumbres y los valores de la comunidad. Para su desarrollo se aplicaron metodologías propias de la innovación social que permitieran el empoderamiento, así como una actitud proactiva de sus integrantes desde los roles que cada uno desempeña.

Este artículo comprende una breve descripción de la comunidad, sus particularidades y situación actual, la revisión de conceptos de innovación social, el desarrollo de procesos, estructuras y liderazgos, el detalle de la situación antes del inicio del acompañamiento empresarial, el proceso llevado a cabo y la situación luego de la incorporación de los resultados del trabajo de campo. Finaliza con la exposición de las conclusiones que permitan dar continuidad al proyecto.

${ }^{4}$ Corregimiento correspondiente al municipio de San José del Fragua, ubicado a 90 kilómetros de Florencia, capital del departamento de Caquetá, en el sur de Colombia. 


\section{Marco teórico y referencial}

\subsection{Contexto de la comunidad}

JAtNEI DtONA o «tabaco huérfano» es una comunidad indígena que vivió los efectos del desplazamiento y, con ello, la desagrupación de sus integrantes. Está ubicada en el departamento de Caquetá, corregimiento de la Fragüita, al margen del río Fragua Chorroso y cerca al Portal del Fragua, lugar que tiene un fuerte significado religioso, ya que lo consideran la génesis de la existencia de esta comunidad. Son descendientes de los Atofe Murui, comunidad ancestral que actualmente se conoce como «uitotos», tradicionalmente asentados en el sur de la Amazonía (Amazonas, Putumayo y Caquetá), región que cuenta con más 6000 indígenas (DANE, 2005). En la actualidad, los JAtNEI DtONA son 76 personas, pertenecientes a 22 familias que llevan seis años en un proceso de asentamiento que se ha facilitado por su cercanía con Florencia, capital del departamento, y a San José del Fragua, cabecera municipal —municipios ubicados a 90 y 40 kilómetros, respectivamente- - unidos por la carretera marginal de la selva que bordea todo el piedemonte amazónico.

La economía es de autosostenimiento, derivada de actividades que han sido tradicionales: la caza y la pesca realizadas por los hombres, y la agricultura desarrollada por las mujeres. Con estas actividades se provee a la comunidad de los insumos necesarios para la preparación de alimentos, especialmente de aquellos derivados de la yuca. No existe ninguna actividad comercial salvo aquellas que se realizan bajo la modalidad de trueque, en especial con otras comunidades indígenas, con las cuales, más allá de un valor monetario, otorgan a los bienes un valor de intercambio dependiendo de las necesidades y la existencia de estos.

En cuanto a la estructura jerárquica, en la comunidad existe un gobernador, máxima autoridad en aspectos organizacionales y económicos; un cacique, líder espiritual de la comunidad; una vicegobernadora, única mujer dirigente en la comunidad — pareja del gobernador-; un secretario, un fiscal y un tesorero.

Una de las principales preocupaciones de la comunidad es la preservación de su cultura. En este sentido, realizan - a través del afianzamiento de la lengua tradicional de los uitotos, del sostenimiento de la tradición oral y de los bailes que son transmitidos en el «círculo de la palabra»- un rito al finalizar cada día con el fin de informar de las actividades llevadas a cabo y proyectar las del siguiente. En complemento con esto, los niños asisten a una escuela gubernamental ubicada a menos de $500 \mathrm{~m}$ de la maloka, lugar de encuentro de la comunidad.

Para el tratamiento de enfermedades utilizan la medicina tradicional indígena, con medicamentos que extrae de la naturaleza uno de los miembros de la comunidad, quien, en compañía del cacique, atiende a los pacientes, esto es, a los habitantes, integrantes de otras comunidades o turistas que llegan hasta el asentamiento en búsqueda de remedio para sus dolencias.

\subsection{Innovación social}

El término innovación social no es nuevo. De hecho, hace más de 4000 años Zaratustra esgrimió una combinación entre dos términos: «la sociedad ideal» y la «mentalidad progresiva», base de lo que en la actualidad conocemos como «innovación social» (Abreu y Jafarey, 2010). Sin embargo, este término ha cobrado gran importancia en las últimas décadas debido a las problemáticas globales que han suscitado mayor atención, tales como la salud, la educación, la inequidad y el medio ambiente (Parada, Ganga y Rivera, 2017). Estas han sido abordadas a nivel global por la Organización de Naciones Unidas (ONU) a través de los Objetivos de Desarrollo Sostenible (ODS), adoptados por todos los Estados miembros 
de la Naciones Unidas en el 2015 «como un llamado universal para poner fin a la pobreza, proteger el planeta y garantizar que todas las personas gocen de paz y prosperidad para 2030» (PNUD, 2015).
Así las cosas, el concepto de innovación social puede abordarse desde distintas perspectivas, teniendo siempre como base la novedad de la actividad y su carácter social tanto en fines como en medios. En la tabla 1 se muestran las diversas aproximaciones que, desde lo teórico, se pueden realizar a su definición.

Tabla 1. Enfoques para el entendimiento de la innovación social

\begin{tabular}{|l|l|l|}
\hline \multicolumn{1}{|c|}{ Enfoque } & \multicolumn{1}{|c|}{ Concepto de innovación social } & \multicolumn{1}{|c|}{ Autores } \\
\hline Pragmático & $\begin{array}{l}\text { Actividades y servicios innovadores que están motivados por el } \\
\text { objetivo de satisfacer una necesidad social y se desarrollan y difunden, } \\
\text { predominantemente, a través de organizaciones cuyos propósitos } \\
\text { principales son sociales. }\end{array}$ & $\begin{array}{l}\text { Mulgan, Tucker, Ali y Sanders } \\
\text { (2007) }\end{array}$ \\
\hline Sistémico & $\begin{array}{l}\text { Proceso complejo a través del cual se introducen nuevos productos, } \\
\text { procesos o programas que conducen a un cambio profundo en las } \\
\text { rutinas diarias, las corrientes de recursos, las relaciones de poder o los } \\
\text { valores dentro del sistema afectado por la innovación. }\end{array}$ & Westley y McConnell (2010) \\
\hline Gerencial & $\begin{array}{l}\text { Nueva solución a un problema social que es más eficaz, eficiente, } \\
\text { sostenible o más justo en comparación con las soluciones existentes, } \\
\text { y que genera valor, principalmente, para la sociedad en lugar de } \\
\text { individuos u organizaciones individuales. }\end{array}$ & Phills, Deiglmeier y Miller (2008) \\
\hline Crítico & $\begin{array}{l}\text { Proceso de empoderamiento y movilización política que apunta a a } \\
\text { una transformación ascendente del funcionamiento de un sistema } \\
\text { social, en términos de partes interesadas y de distribución de recursos } \\
\text { materiales e inmateriales. }\end{array}$ & $\begin{array}{l}\text { Maccallum, Moulaert, Hilliery } \\
\text { Vicari (2009) }\end{array}$ \\
\hline Económico & $\begin{array}{l}\text { Cambio conceptual, de proceso o producto, organizacional o en el } \\
\text { financiamiento. Nuevas relaciones con partes interesadas y territorios. }\end{array}$ & OCDE (2006). \\
\hline Comparativo & $\begin{array}{l}\text { Distinción de los resultados, las nuevas relaciones, las nuevas formas } \\
\text { de cooperación yaportes, así como de los procesos, métricas, modelos } \\
\text { y métodos utilizados en la innovación en los campos comerciales o } \\
\text { tecnológicos, no siempre transferibles a la economía social. }\end{array}$ & $\begin{array}{l}\text { Murray, Caulier-Grice y Mulgan } \\
\text { (2010) }\end{array}$ \\
\hline Universal & $\begin{array}{l}\text { Soluciones - productos, servicios, modelos, mercados, procesos, } \\
\text { etc.- que satisfacen de manera simultánea una necesidad social } \\
\text { - de forma más eficaz que las soluciones existentes-y conducen } \\
\text { a capacidades y relaciones nuevas o mejoradas y a un mejor uso de } \\
\text { activos y recursos. }\end{array}$ & The Young Foundation (2012) \\
\hline
\end{tabular}

Fuente. Elaboración propia a partir de Balamatsias, 2018.

El Manual de Oslo (OCDE y Eurostat, 2006) sugiere que, a fin de que la innovación social sea real, lo más relevante es su utilización efectiva y continuada, traducida en apropiaciones sociales a través del uso. Coincide con el enfoque de Hernández, Tirado y Ariza (2016), quienes explican cómo los usuarios serían fuentes de innovación social al emplear las innovaciones introducidas en su vida cotidiana.

A partir de lo anterior, y en consideración a que la innovación social no es un hecho puntual sino que obedece al cumplimiento de una serie de etapas que se articulan para la obtención de los fines propios de su implementación, el modelo de innovación social propuesto por Murray et al. (2010), adaptado por Buckland y Murillo (2014), plantea que el proceso de innovación social se compone de seis etapas que deben servir como marco de referencia para el planteamiento de proyectos de este tipo, en las cuales, además, se requiere de las habilidades y los conocimientos provenientes de distintas disciplinas y saberes. Estas etapas son las que se describen a continuación:

- Diagnóstico. Supone la identificación del entorno en el que se plantea el proyecto, de las distintas partes interesadas en su desarrollo y del problema 
a solucionar, así como su origen. Es una etapa en la cual se debe resaltar el reto de innovación que sirva como el agente motivador para la búsqueda de diferentes alternativas de solución.

- Propuestas e ideas. Es la etapa de mayor creatividad de todo el proceso. En ella se debe lograr la formulación de la mayor cantidad de soluciones sin cuestionar su posibilidad de implementación; no es un momento para la determinación de la calidad de las ideas, es conveniente dar libertad a la proposición de soluciones.

- Prototipos y pilotos. En esta etapa se realiza la valoración de las ideas al probarlas de diversas maneras, iterar los resultados y medir sus impactos, a fin de escoger aquella solución que satisfaga de mejor manera y sea la más pertinente para la atención de las necesidades de la población a la cual se dirige. Esta actividad elimina la incertidumbre en la misma medida en que se lleva a cabo atendiendo a las condiciones naturales del entorno.

- Modelo de negocio y sostenibilidad. Con la puesta en marcha de esta etapa el prototipo se lleva a la realidad, de modo que se definen las modalidades de generación de ingresos que permitan el mantenimiento de la iniciativa en el largo plazo.

- Escalamiento y difusión. Se puede denominar la etapa de madurez de la innovación. En ella se procura que la estrategia se disemine e implemente en escenarios distintos al original, hecho que permite la validación total de la iniciativa propuesta, con lo cual se contribuye al éxito y se conservan las características iniciales del proyecto, pero adaptándolo en los casos en los cuales así se requiera.

- Cambio sistémico. En esta fase, con objetivos de largo plazo, se busca que la interacción se dé a gran escala, con movimientos sociales que, además de facilitar la difusión de las ideas, las formas de pensar y las actividades propias de la iniciativa también sirvan como sus dinamizadores y propulsores en diferentes ecosistemas.

Este modelo, adaptado por Buckland y Murillo (2014) para el contexto latinoamericano y complementado con el modelo de diseño del diamante doble del Design Council (2007), ha dado base a la metodología del doble hexágono (Jiménez, 2017), usada en el proceso de intervención en comunidades indígenas con la inclusión de herramientas pertinentes para su contexto (Patiño, Ruiz y Echeverry, 2018).

Con este modelo se busca que exista la figura de un facilitador que, más allá de un consultor, basado en el enfoque de pensamiento de diseño logre poner en el centro del proceso a las personas $y$, a partir de su participación, desarrolle un proceso de innovación del que resulte la proposición de alternativas que sirvan como solución a problemas u oportunidades que se presenten en la comunidad a acompañar, debidamente validadas e implementadas.

Con la adaptación realizada, el nuevo modelo se visualiza en la figura 1 . 
Figura 1. Modelo para la innovación social adaptado

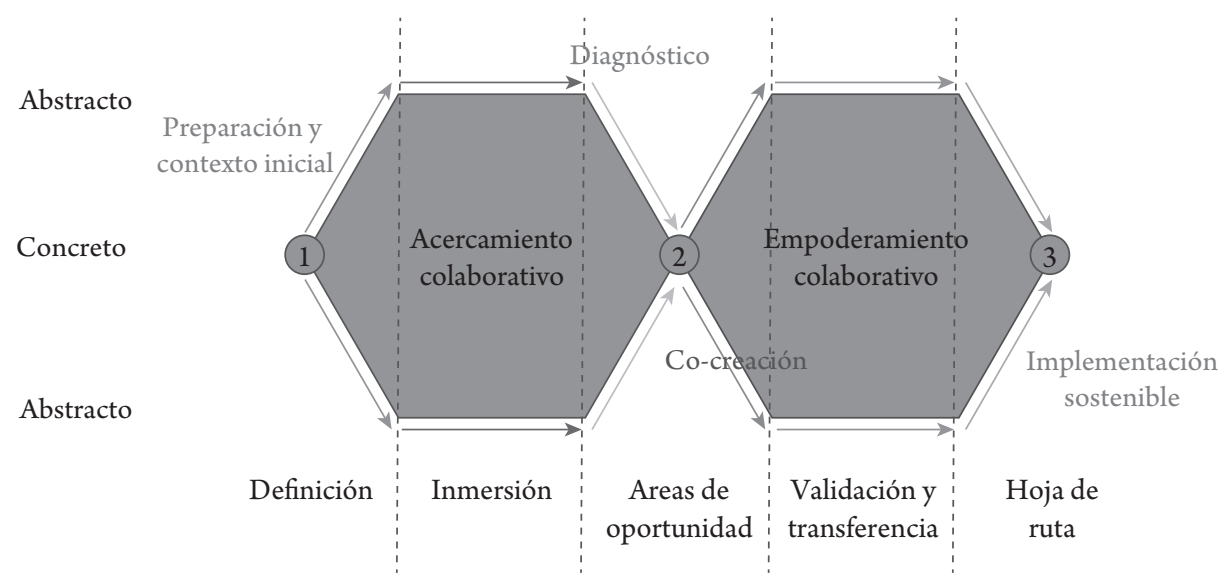

Fuente. Patiño et al., 2018.

\subsection{Empresa social y emprendedores sociales}

La empresa social, antes asimilada a las organizaciones sin ánimo de lucro, ha evolucionado. Su concepto es ahora más amplio, tal como lo plantea Social Enterprise Alliance (2019), organización que la define como un tipo de organización que, con base en el enfoque de mercado, busca satisfacer una necesidad básica no satisfecha o desarrollar soluciones a problemas sociales o ambientales. Esta concepción, que no es excluyente y la pueden trabajar organizaciones con o sin ánimo de lucro, además de contribuir con causas sociales tiene como una de sus fortalezas la generación de ingresos que permitan asegurar la sostenibilidad futura de las iniciativas mediante la obtención de resultados financieros que permitan a los emprendedores sociales un retorno sobre su inversión o a las organizaciones sin ánimo de lucro la reinversión de sus excedentes en el crecimiento de la iniciativa o en la atención a nuevas necesidades. El modelo, así planteado, se concibe desde una visión integral según la cual este tipo de organizaciones logra la suma del propósito social con el valor empresarial, involucrando la disciplina, la innovación y la determinación propias de la empresa con ánimo de lucro.

Así las cosas, con esta forma de entender la innovación social se busca generar la creación de empresas sociales, entendidas como organizaciones de categoría híbrida que cumplen un doble propósito de creación de valor social y de sostenibilidad empresarial (Alter y Dawans, 2006). En este contexto, el proyecto busca empoderar a la comunidad con el fin de que sus integrantes oficien como emprendedores sociales e individuos que desarrollan una innovación social y crean una empresa social con base en ella (Phills et al., 2008).

\subsection{Diseño participativo centrado en las personas}

Desde la perspectiva de la investigación-acción participativa, Ander-Egg (2003) define que una metodología de intervención de carácter participativo implica que las personas involucradas intervengan en el estudio y diagnóstico de su realidad, en la definición de las soluciones a desarrollar y en la forma de desarrollarlas. Esto se logra con pautas y elementos técnicos y operativos que favorecen la participación y hacen que ella sea posible y efectiva.

Estos mismos principios se observan también desde la perspectiva del diseño en el marco de la investigación generativa, los cuales se han desarrollado e implementado en diferentes campos a través de la implementación de metodologías que buscan involucrar a los sujetos de investigación como parte activa del proceso de diseño de soluciones que inicia con el claro entendimiento de un problema (Sanders y Stappers, 2012). 
El diseño se transformó desde finales del siglo XX cuando pasó de enfocarse solo en el diseño de productos a solucionar problemas complejos mediante el desarrollo de metodologías como, por ejemplo, el pensamiento de diseño, adoptado por el sector empresarial para generar soluciones estratégicas y diseñar nuevos modelos de negocio, llegando también a aplicarse en Organizaciones no Gubernamentales (ONG) o empresas sociales con el fin de desarrollar soluciones más pertinentes a problemas sociales (Brown y Wyatt, 2010). El pensamiento de diseño se fundamenta en el descubrimiento de hallazgos significativos profundos delos usuarios o personas involucradas en el problema a solucionar, así como en un proceso experimental en el cual se prototipan soluciones a fin de estar en capacidad de recibir retroalimentación directa de estas mismas personas y evitar de esta manera basarse en suposiciones sobre lo que puede ser el problema o la necesidad (Brown y Wyatt, 2010).

Por otra parte, es un proceso entendido como un sistema de espacios que se traslapan, más que una serie de fases lineales; estos espacios son: inspiración — problema u oportunidad que motiva la búsqueda de soluciones-, ideación — proceso para generar, desarrollar, prototipar y testear ideas- e implementación — la ruta que guía la solución desde el proyecto hacia la vida de las personas-.

IDEO (2015), empresa consultora pionera en el desarrollo e implementación de estas metodologías en el campo del diseño, define en su guía de campo el diseño centrado en las personas - human centered design - como «un proceso y un conjunto de técnicas que se usan para crear soluciones - a nivel de productos, servicios, espacios, organizaciones y modos de interacción- nuevas para el mundo» (p. 6). Este proceso está centrado en las personas que hacen parte del contexto, viven de primera mano el problema que se quiere resolver y se involucran en todas las etapas del proceso que inicia con la identificación de las necesidades más profundas de las personas, entendidas desde su origen —más allá de lo observable - y que definen así la dimensión de lo que es deseable. En las siguientes fases del proceso se involucran otras dos dimensiones: la factibilidad —si la solución se puede desarrollar desde la tecnología y el contexto- y la viabilidad — si la solución funciona a nivel sistémico o de modelo de negocio-. Esto facilita el proceso real de innovación que es la implementación real de las soluciones en los mercados y las comunidades (IDEO, 2015). Los momentos de ideación e implementación en el pensamiento de diseño, al involucrar también a las personas como expertos de su propio contexto y problema y basarse en procesos de experimentación, facilitan la evaluación de viabilidad y la factibilidad, de modo que se disminuyen factores de riesgo para su sostenibilidad.

Inglesis-Barcellos y Botura (2017) han planteado que el pensamiento de diseño tiene como uno de sus elementos esenciales y estratégicos la participación del talento humano. En tal sentido, Barab et al. (2002) han introducido el concepto de «diseño de empoderamiento», según el cual se trabaja con el pensamiento de diseño aplicado a desarrollos en grupos de trabajo con el fin de que los individuos transformen sus prácticas, introduzcan cambios en su cultura y luchen por la construcción de un mundo mejor. Lo anterior a pesar de las condiciones de intervención que, la mayoría de veces, exigen un compromiso multidisciplinar por el cual se agrupen agendas que pueden ser conflictivas entre los miembros de una colectividad.

Por otra parte, el empoderamiento en las iniciativas de carácter social tiene dos caras. La primera que se muestra es la del emprendedor que la propone y la segunda la de la comunidad que recibirá los beneficios de ella. En este sentido, el empoderamiento del primero se da por descontado dado que es parte esencial de su iniciativa, pero en el caso de la segunda es uno de los grandes retos a afrontar dado que en la misma medida en que se logre se facilita el éxito del proyecto por la apropiación que los beneficiarios hacen de él. 


\section{Antecedentes}

$\mathrm{A}$ pesar de que los fines no eran comerciales o lucrativos, la comunidad JAtNEI DtONA, antes del proceso de acompañamiento, desarrollaba procesos de producción y transformación de materia prima cuyo resultado son productos que satisfacen las necesidades de subsistencia de sus integrantes o son parte de su cultura, en la cual juega un papel importante la realización de los rituales y las ceremonias.

Algunos procesos de producción se realizan de manera permanente - p. ej., ambil, mambe, casabe y ají- y otros de manera esporádica - p. ej., jabón y artesanías-; estos últimos con el fin de comercializarlos en ferias y eventos municipales.

Los procesos productivos están muy arraigados con la cultura y las costumbres de la comunidad. El ambil, una pasta negra obtenida a partir de la cocción del tabaco mezclado con diferentes sales naturales, lo usan los miembros de la comunidad en la maloka en el momento del círculo de la palabra — sin distinción de sexo- - Por su parte, el mambe, polvo obtenido por la reducción de la coca mezclada con algunas cenizas provenientes de otros vegetales, se usa en ese mismo círculo, pero solo lo producen y consumen los hombres. Tanto el ambil como el mambe se consideran elementos sagrados en la cultura uitoto, en la medida en que proveen sabiduría para la toma de decisiones.
Por otro lado, producen harina de yuca (casabe) que consumen en distintas preparaciones autóctonas, elaborada por las mujeres con la colaboración de los hombres solo para la labor de cernido, así como ají, el cual hace parte esencial de los hábitos de consumo alimenticio de la comunidad. Por último, entre los productos elaborados se encuentran el jabón —que no es una tradición de la comunidad sino una actividad desarrollada de manera muy puntual por dos mujeres de la comunidad que usan insumos naturales para su producción-, y las artesanías cuya producción no es sistematizada y se da de manera esporádica por parte de las mujeres de la comunidad.

Es importante aclarar que las actividades diarias de la comunidad, en su gran mayoría, las realizan mujeres, dejando que los hombres se ocupen tan solo de la caza, la pesca y la elaboración de insumos sagrados. Las actividades productivas y agrícolas las desarrollan mujeres con la participación esporádica de algunos hombres que colaboran en labores que demandan gran esfuerzo físico.

Tener estas actividades sin que ellas fueran consideradas como una forma de generación de recursos para la comunidad fue el motivo por el cual algunos de los miembros vieron una posibilidad de estructuración de un proyecto productivo, para lo cual se acercaron a la Universidad Ean y su programa Ean Impacta. 


\section{Proceso de acompañamiento a partir de la metodología del doble hexágono}

\subsection{Fase 1: Preparación y contexto inicial}

El objetivo de esta fase fue alinear los objetivos y los alcances del proyecto, validar información del contexto inicial y determinar acuerdos orientados al primer encuentro con la comunidad. Así mismo, probar herramientas y métodos para el trabajo de campo, definir el equipo y el plan de trabajo.

En el caso que se presenta, la primera fase se realizó con la participación de personas y organizaciones que han desarrollado proyectos de acompañamiento con comunidades indígenas o trabajan de manera permanente con ellas, entre las cuales se encuentran la Agencia Nacional para la Superación de la Pobreza Extrema (Anspe). El trabajo introductorio llevó al conocimiento y al entendimiento de costumbres $y$ códigos de comportamiento y uso del lenguaje que podrían facilitar el desarrollo de la visita.

Por otra parte, se realizaron reuniones de aproximación a la comunidad - se muestran en la figura 2 - en las cuales se establecieron los compromisos de las partes y las actividades a realizar en la primera visita a campo.

Figura 2. Sesión precampo

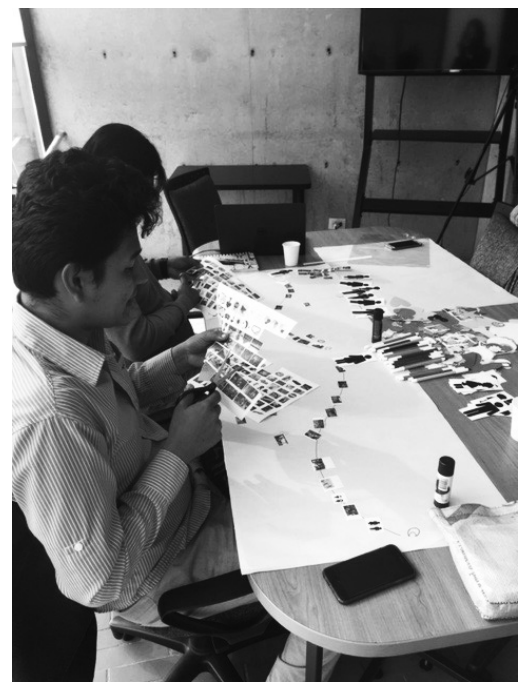

Fuente. Elaboración propia.

\subsection{Fase 2: Acercamiento colaborativo - visita de campo 1 -}

El propósito de esta fase fue realizar una inmersión en el contexto de la comunidad por medio de investigación de campo con métodos etnográficos y centrados en las personas, a fin de identificar de manera clara y cercana la situación, los actores, sus relaciones y las necesidades de la comunidad. Algunos de los métodos utilizados fueron: cartografía social - actual y futura-, tour guiado, mapa de actores y relaciones, y un día en la vida de... en tres años. La figura 3 muestra la representación que hacen de la situación geográfica actual de la comunidad. 
Figura 3. Resultado de ejercicio cartografía social

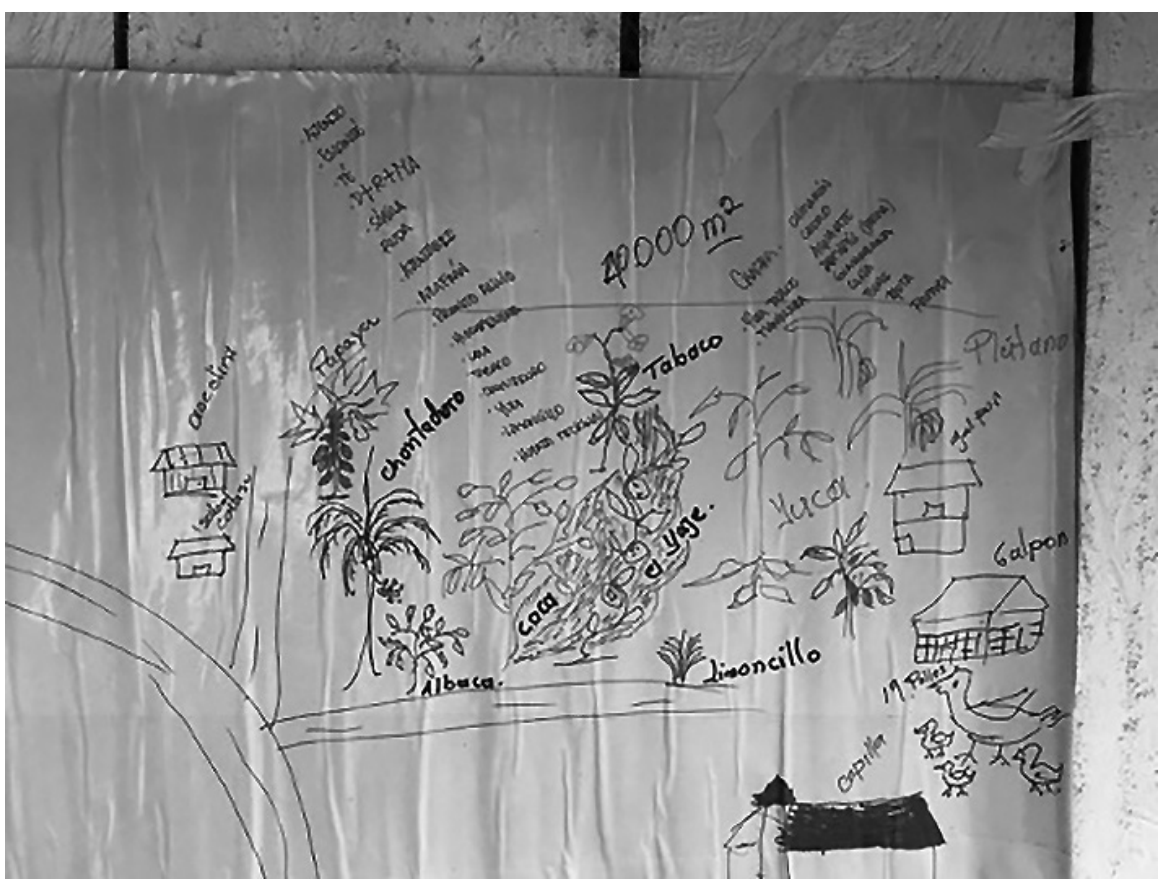

Fuente. Elaboración propia.

Con el uso de estas herramientas se logró establecer de manera clara el rol de cada uno de los participantes en la comunidad, sus responsabilidades y las actividades que realizan. En la sesión se contó con la participación activa de 37 miembros de la comunidad, más del $50 \%$ de esta y la pasiva del gobernador y del alcalde, quienes hicieron presencia, pero solo en calidad de observadores del proceso. Parte del éxito del proyecto radicó en el uso de metodologías de diseño participativo que permitieron a la comunidad ser parte del diseño desde el momento cero del proyecto. La participación activa de la comunidad permitió hacer una reflexión sobre la situación actual y abrir oportunidades a nuevas formas de pensar $y$ hacer.

\subsection{Fase 3: Diagnóstico — visita de campo 1 -}

En esta fase se analiza toda la información recolectada, se generan conclusiones y se producen hallazgos que luego se presentan a la comunidad a fin de validarlos. Se desarrolla en los siguientes pasos:
- Sensibilización concepto de emprendimiento sostenible

- Reflexión sobre actividades realizadas

- Conclusiones proyectos con potencial de sostenibilidad

Dado que el objetivo del proyecto fue la identificación de las diferentes alternativas para desarrollar actividades productivas que permitieran la generación de recursos, sin que esto afectara los patrones culturales de la comunidad, esta fase de trabajo se realizó con miras a las actividades que, de manera regular, se realizan en la comunidad (Figura 4). En este sentido, se identificaron cinco posibles productos a elaborar con potencial para venderse en el mercado. Dichos productos son: ambil, mambe, artesanías elaboradas con productos de la región, productos orgánicos de aseo - jabón y champú- y ají ahumado en polvo. 
Figura 4. Resultado actividad proyectos potenciales
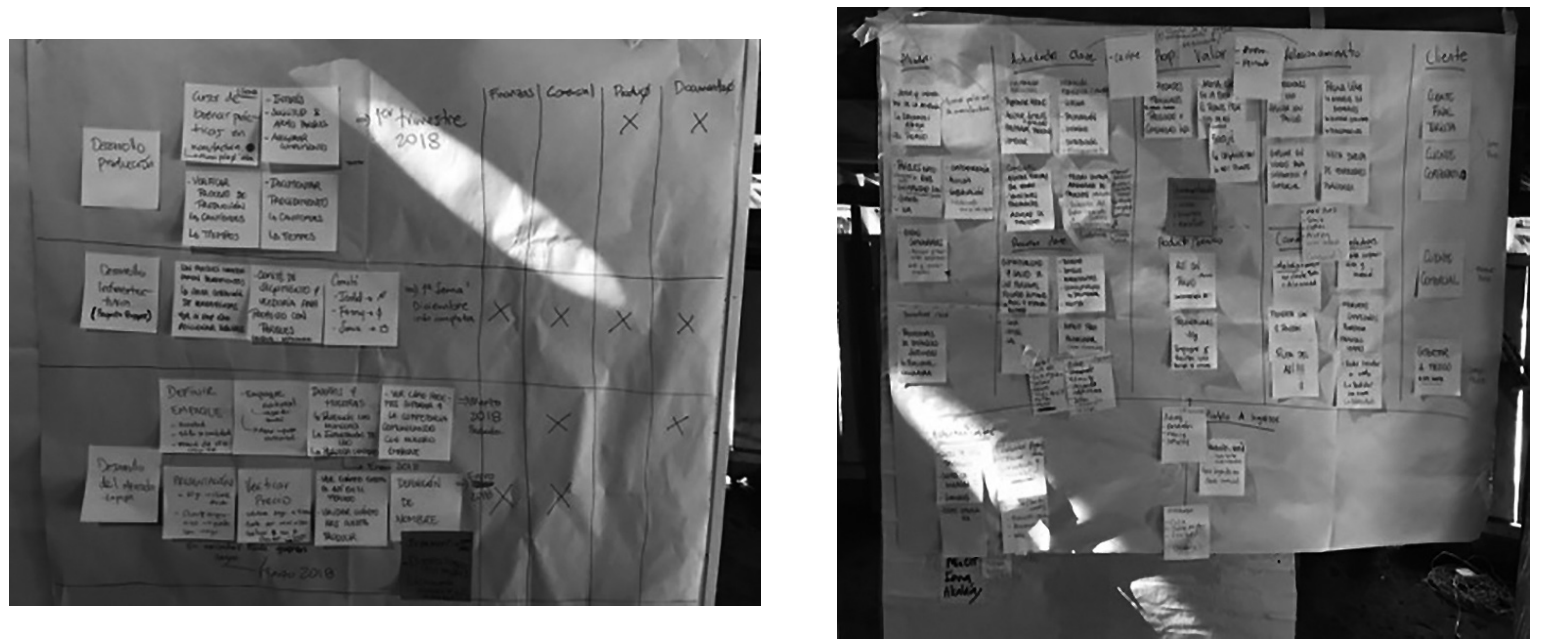

Fuente. Elaboración propia.

\subsection{Fase 4: Cocreación de alternativas - visita de campo 1 -}

En esta etapa se requiere el trabajo conjunto entre los facilitadores y la comunidad. Tanto unos como otros proponen modelos que se ajustan a las potencialidades de la comunidad, las vocaciones y las motivaciones personales, definen áreas de oportunidad según proyectos identificados como potenciales en fases anteriores, diseñan modelos de negocio (Board of Innovation, s. f.) y profundizan en las diferentes alternativas del modelo de negocio, tal como se muestra en la figura 5 .

Figura 5. Cocreación con la comunidad JAtNEI DtONA
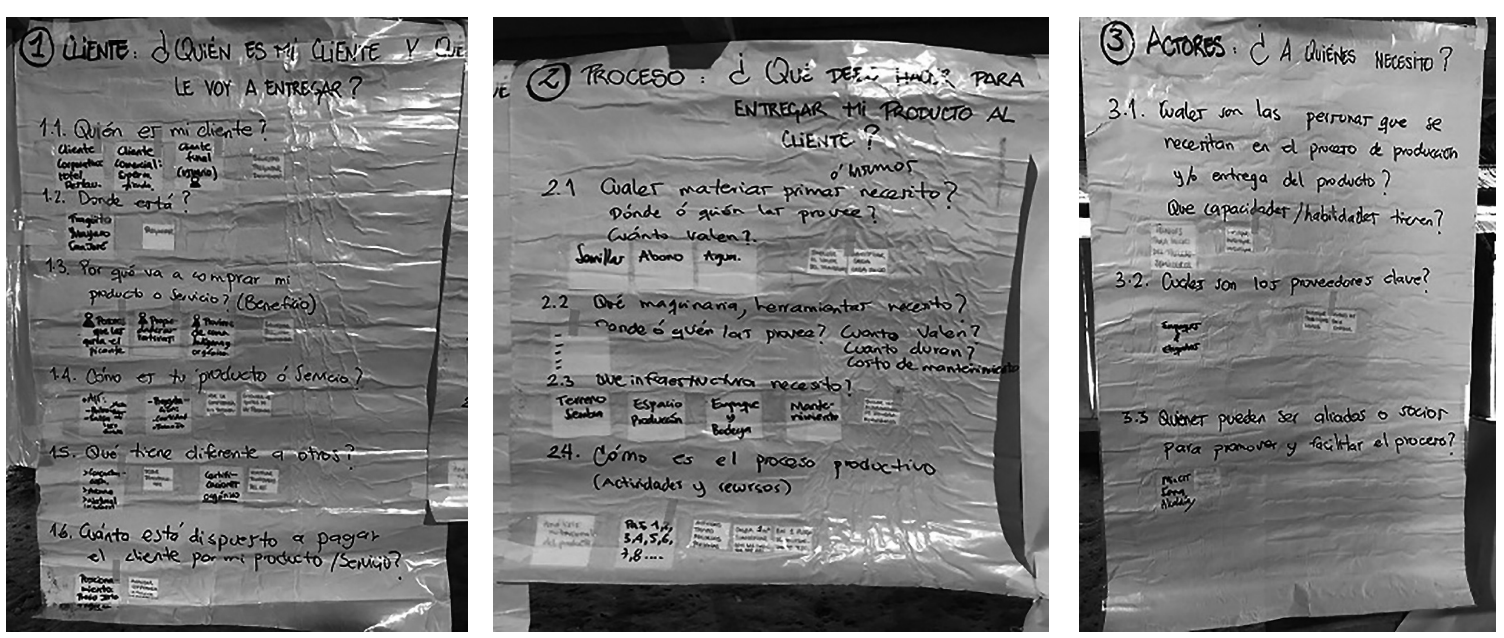

Fuente. Elaboración propia. 
Esta fase se realiza mediante la formulación de preguntas para validación — prácticas para el desarrollo de modelos de negocios creativos basados en el desarrollo de preguntas- (Richard, 2013) y la definición de equipos de trabajo y pasos a seguir en el desarrollo del modelo de negocio. El resultado de esta etapa se muestra en la figura 6.

Figura 6. Construcción del modelo de negocio, con la comunidad JAtNEI DtONA

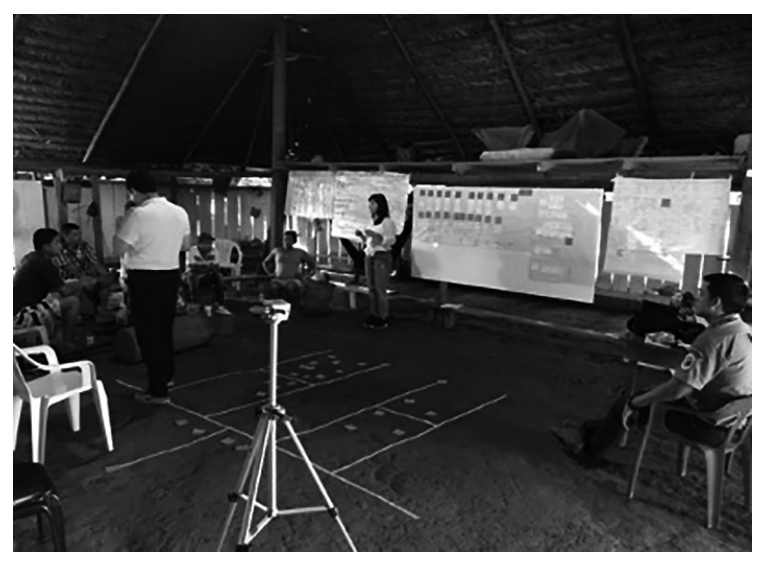

Fuente. Elaboración propia.

Con el fin de establecer la primera línea de acción para la generación de recursos propios, la comunidad escogió el ají, artículo con el que la comunidad ya tenía algunas experiencias de intercambio no monetario con otras comunidades. La producción de ají era, hasta el momento de la primera visita, una actividad que se encontraba integrada a sus costumbres alimenticias, pues la siembra y la cosecha de este condimento se realizaba con el propósito de suplir las necesidades de la comunidad. Esto generaba unos pocos excedentes que servían, además del trueque, para pequeñas ventas a turistas que visitaban la maloka.

La escogencia realizada por los indígenas, con el acompañamiento del grupo de investigadores, atendió a situaciones del entorno. El primer reto en el proceso fue lograr que de una concepción de autosubsistencia se pasara a una de negocio con la cual fuera posible obtener ingresos que ayudaran al mejoramiento de las condiciones de vida de todos los integrantes, así como en el cumplimiento de los sueños que algunos de ellos identificaron en el desarrollo de las distintas actividades. De esta manera, un primer logro fue

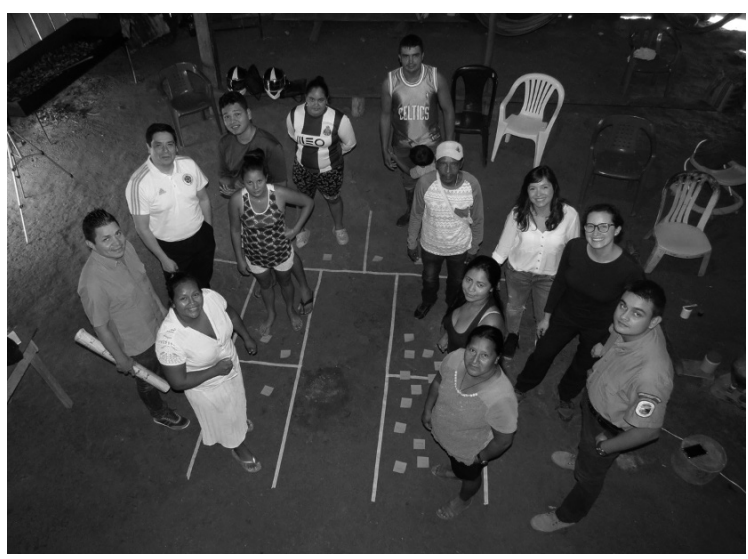

que los indígenas, principalmente las mujeres y los jóvenes, visualizaran un futuro más allá de la maloka, que vislumbraran distintas opciones; por tanto, la motivación fue el primer factor que facilitó el desarrollo del proceso.

Con esta fase se culminó la primera visita de campo, con lo cual se estableció que la comunidad, en el desarrollo de la iniciativa, debía adelantar tareas puntuales, algunas relacionadas con el proceso productivo y otras con la organización de tareas. Estas actividades se enlistan a continuación.

En la producción:

- adecuación del terreno para la siembra

- germinación de las semillas

- plantación de las semillas

- desarrollo de abono

En lo empresarial:

- asignación de roles y responsabilidades 


\subsection{Fase 5: Empoderamiento colaborativo - trabajo autónomo y visita de campo 2 -}

Este fue el momento para validar y fortalecer los proyectos propuestos y establecer compromisos que permitieran a la comunidad empoderarse de los proyectos e implementarlos sin depender de actores externos - transferencia de conocimiento-. Las estrategias metodológicas que se usaron en esta etapa fueron:

- socialización, investigación y análisis desarrollado

- blueprint de proceso de producción, etapas, recursos, costos, personas involucradas y nuevas preguntas

- especificación de proyecto y hoja de ruta

La segunda parte del proyecto se realizó tres meses después, verificando los avances de la comunidad y el cumplimiento de las actividades que se recomendaron en el final de la primera visita. Estos avances son los siguientes:

En la producción:

- delimitación clara del área a cultivar

- adecuación del terreno, con su respectivo sistema de riego

- siembra de las plantas de ají — para la primera fase de crecimiento se creó un pequeño invernadero con materiales reciclados-

- diseño del primer prototipo de empaque
En otros aspectos empresariales:

- determinación de una estructura básica de costos

- investigación de posibles compradores

- división de roles y actividades en el interior de la comunidad

- empoderamiento de los miembros de la comunidad en proceso total de producción y comercialización

\subsection{Fase 6: Implementación sostenible - visita de campo 2 y monitoreo-}

La fase final del proceso busca garantizar la permanencia del modelo de negocio, por lo cual es necesario que se desarrolle la estrategia de sostenibilidad, de manera detallada, con herramientas como, por ejemplo, el modelo canvas, entre otros. En esta fase se identifican oportunidades y se ponen en marcha actividades en aspectos que ayudan a la consolidación tales como branding, comunicación, formalización de la estructura organizacional y determinación de cronogramas e hitos empresariales a alcanzar.

Con la comunidad a la cual se ha hecho el acompañamiento no se ha llegado a esta fase de desarrollo, dado que se trata de aspectos que solo se pueden lograr en la misma medida que se tenga un modelo de negocio maduro. El proceso se lo desarrolla la comunidad y los resultados dependen de que ellos mantengan el nivel de compromiso y empoderamiento que han logrado durante las fases previas, a pesar de que ya existan avances verificables de la implementación del modelo de negocio (Figura 7). 
Figura 7. Semillero de ají, construido por la comunidad JAtNEI DtONA

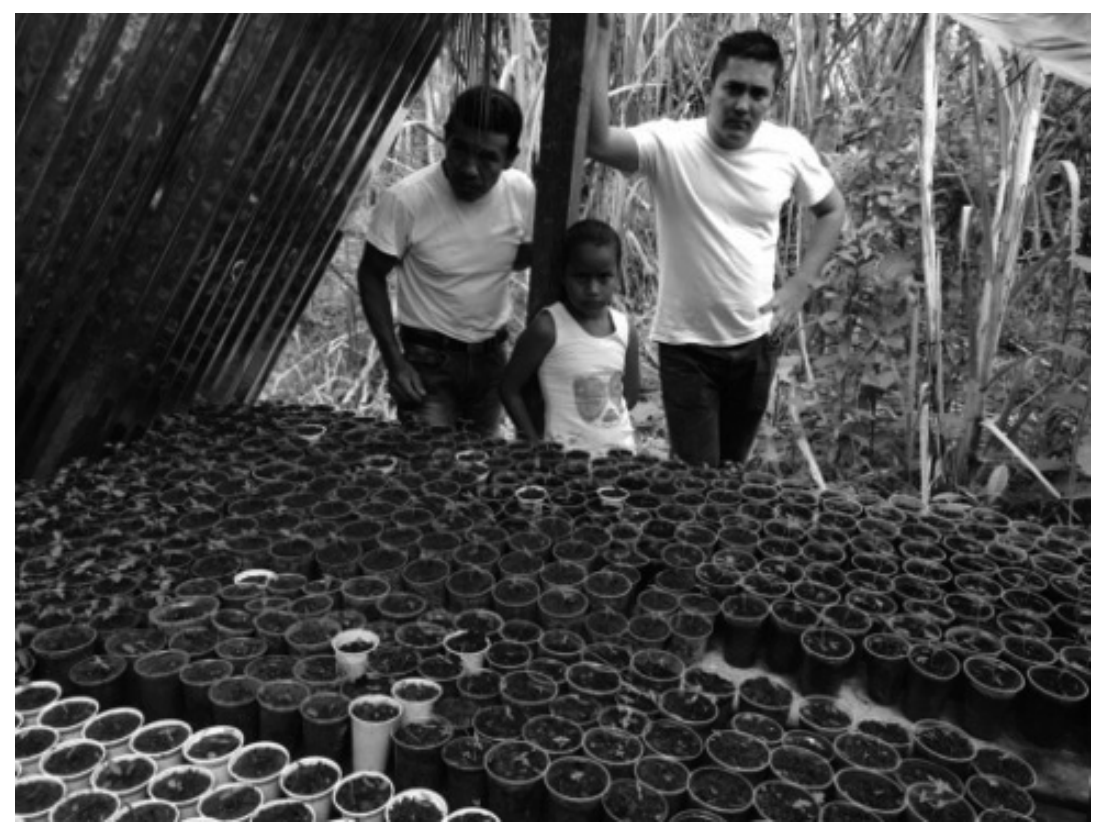

Fuente. Elaboración propia.

Sibien el proceso de implementación de los resultados de innovación social es de largo plazo y de una amplia cobertura en ecosistemas, el trabajo realizado con la comunidad JAtNEI DtONA apenas ha alcanzado el cumplimiento de las cuatro primeras fases del proceso sugerido por Murray et al. (2010) y las cinco primeras según el modelo del doble hexágono.

Sin embargo, la metodología propuesta permitió empoderar a la comunidad y dar frutos sin haber culminado todas las etapas. En este tipo de procesos de innovación social es clave empoderar a la comunidad desde el inicio mediante el fomento de la participación de todos a fin de lograr los objetivos de la autoorganización para obtener acceso a recursos y de la inclusión de procesos organizativos y estructuras que permitan el desarrollo de una iniciativa económica para el mejoramiento de la calidad de vida de los miembros. El empoderamiento precisamente es esa acción colectiva que permite mejorar la calidad de vida en una comunidad (Zimmerman, 2000).

Al final de esta primera fase de acompañamiento la comunidad identificó su potencial productivo y acopió los recursos físicos, financieros y humanos para el inicio del proyecto. Una hectárea de terreno se preparó para el cultivo del ají y cerca de 1000 semillas se sembraron con el propósito de obtener los cogollos que luego serían llevados a la tierra preparada, con lo cual pueden proyectar la producción y la comercialización en los siguientes cuatro meses, tiempo en el cual se puede hacer la primera cosecha y procesar las primeras unidades para la venta. 


\section{Hallazgos}

$\mathrm{E}$ objetivo inicial del proyecto fue formular alternativas para la generación de recursos en la comunidad indígena escogida, sin embargo, los hallazgos en el proyecto van más allá, dado que se evidenciaron particularidades en la cultura y las costumbres de la comunidad que permiten encontrar otros elementos no contemplados en el inicio y que son esenciales para el desarrollo de estas iniciativas, tales como la distribución de funciones entre hombres y mujeres, o el liderazgo ejercido por algunas de ellas a pesar de no ser parte de la estructura jerárquica de la comunidad, entre otros.

Las herramientas generativas y participativas, basadas en el diseño centrado en las personas, permitieron vincular a la comunidad con el proyecto durante todas las fases desarrolladas hasta el momento, lo que incluye hombres, mujeres, ancianos y niños, al darle igual importancia a las opiniones de todos los integrantes sin ninguna diferenciación por rol, edad o sexo. Esto facilitó la conexión con sus creencias, valores y costumbres a todo nivel. El trabajo articulado de facilitadores y comunidad se realizó alrededor de tres ejes: impacto en las personas, preservación de su territorio y generación de ingresos con una visión que va más allá de la subsistencia.

En cuanto a los impactos en las personas fue importante la identificación de los roles de cada una de las personas en la comunidad y la manera como muchos de ellos potencializaron y cambiaron con el avance del proyecto. En este sentido, el ejercicio del liderazgo cambió de manera progresiva a lo largo del tiempo, sin que esto impactara la estructura organizacional de la comunidad, distinta de la de la iniciativa de negocio. Mientras el gobernador y el cacique mantienen su jerarquía en la comunidad, las ideas de negocio fueron planteadas, iniciadas y potencializadas por otros actores, entre quienes adquirieron un papel muy importante las mujeres de la comunidad, con expectativas distintas y con mayor propensión al emprendimiento, dado que muchas de las actividades son ejecutadas por ellas.

Este liderazgo sirvió como base del empoderamiento que los integrantes de la comunidad han desarrollado con el proyecto. Desde un desconocimiento total de las distintas oportunidades de negocio que existían, pasando por la identificación de los posibles productos a fabricar, se llegó hasta el momento en el cual los interesados en ser partícipes del proceso asumieron responsabilidades frente a este, de tal manera que pasara de ser idea a convertirse en proyecto, todo como producto de la motivación que propició el reconocimiento de sueños que podían hacerse realidad a partir de su expresión en las metodologías implementadas.

Todo lo anterior solo adquiere trascendencia en la misma medida en que los integrantes perciben que todas las actividades no riñen con su cultura y costumbres y, por el contrario, son el instrumento para que se mejore la calidad de vida y se cuente con los recursos necesarios para su preservación, de tal manera que favorezcan a la comunidad en la disminución de su dependencia de recursos oficiales, reducidos o de lo provenientes de otras instituciones, los cuales, casi de manera generalizada, se proveen con un criterio completamente asistencialista.

Frente a las nuevas condiciones, la comunidad identificó la carencia de conocimientos en aspectos técnicos del modelo de negocio. A diferencia de los conceptos empresariales que fueron asimilados por los participantes, los estrictamente técnicos relacionados con la producción del ají generaron necesidad de apoyos de otros profesionales y de capacitación con el fin de tener autonomía futura. Como consecuencia de esto la comunidad debió acudir al acompañamiento de una agrónoma que les enseñó técnicas de cultivo y de cuidado de la siembra, conocimientos que fueron interiorizados por ellos, logrando una importante cualificación del recurso humano. 
El mismo proceso ha hecho que la comunidad aprenda y modifique su forma de operar, con cambios que impactan sobre la estructura organizacional y los aspectos financieros del proyecto. En un comienzo, las actividades las realizaban algunos miembros de la comunidad sin que hubiese un reconocimiento individual por la cantidad y la calidad del trabajo personal, de modo que los ingresos eran comunales, principio aceptado y compartido por todos los miembros de la comunidad.

Las mismas circunstancias hacen que la comunidad desarrolle esquemas de trabajo que hagan más eficientes los procesos, para lo cual establecieron que los ingresos deben ser repartidos entre las personas que ejecutan cuatro funciones básicas: producción, transformación, comercialización y administración, por lo cual corresponde el $30 \%$ a las tres primeras y el restante $10 \%$ a la última. Esto deja claro que existe una división del trabajo y cómo las personas que ejercen cada una de las funciones nombradas pueden tener acceso a una remuneración individual por su trabajo.

Las condiciones para que la iniciativa de negocio sea sostenible en el tiempo ya se están incorporando en la cultura de la comunidad. Así, desde el punto de vista comercial, han participado en varias ferias locales que les han permitido determinar la aceptación que puede tener el producto, no solo entre el canal a través del cual comercializan actualmente - distribuidores mayoristas-, sino también entre restaurantes y el público en general. Con esto pueden lograr un mejor precio y, como consecuencia, una mayor rentabilidad.

\section{Conclusiones}

$\mathrm{L}$ a cultura de una organización y las circunstancias que la rodean generan patrones de comportamiento que llevan a los miembros que la integran a permanecer en un statu quo de aceptación de dichas condiciones, sin vislumbrar la posibilidad de que existen oportunidades más allá de la misma organización.

En este sentido, un proyecto de intervención, en este caso una comunidad indígena que busque identificar posibles ideas de negocio y ponerlas en marcha, debe partir del principio de la disposición de los miembros que integran la organización, lo cual parece ser obvio, pero no lo es tanto en el caso analizado, toda vez que, al ser parte de una comunidad, existen patrones culturales arraigados que pueden dificultar su implementación.

Acorde con lo anterior, la generación de impactos positivos en la comunidad debe ser consecuencia de un proceso en el cual las personas, en su individualidad, comprendan e incorporen los beneficios que puede traer, para ellos en particular y para la comunidad en general, la generación de ingresos que permiten el mejoramiento en las condiciones de vida.
En este sentido, aspectos relacionados con la motivación, el empoderamiento y una visión de futuro individual que pueda ser común a distintos miembros de la comunidad resulta de vital importancia, más allá de los aspectos técnicos propios de cualquier iniciativa que se pretenda seguir.

No se trata de romper con sus patrones culturales, en los cuales la colectividad es uno de los ejes centrales de cohesión de la comunidad. Se trata de que la comunidad perciba las posibilidades de crecimiento que tiene y que cada uno de los individuos se identifique con ello y vea las posibilidades propias.

En este orden de ideas, la propiedad comunal y el trabajo - las bases fundamentales de su desarrollo social y económico- son elementos clave sobre los cuales cualquier propuesta que tiende a generar ingresos debe estar cimentada, al igual que la identificación de actividades que respeten sus tradiciones, como, por ejemplo, la protección del medio ambiente que les proporciona los alimentos necesarios para su subsistencia o la participación activa de mujeres en procesos productivos, una parte integral de la cultura indígena con la que se realizó el trabajo de investigación. 
No es el objetivo, en ningún momento, introducir a las comunidades en una economía de consumo que no consideren necesaria; el esfuerzo debe dirigirse a elevar la conciencia de que, en medio de su cultura, con total respeto por sus tradiciones, las comunidades pueden generar procesos internos que los lleven a mejorar su vida que condiciona y potencializa su sostenibilidad. Las metodologías de diseño participativo permitieron que nuevas dinámicas sociales ocurrieran dentro de la comunidad JAtNEI DtONA y pudieran nacer emprendedores sociales, lo cual desencadenó en la creación de los inicios de una empresa social.

Esta situación implica que necesitan orientación especial a fin de ayudarles a identificar alternativas que permitan generar sostenibilidad para estas comunidades recién nacidas. Tales condiciones

\section{Referencias}

Abreu, J.; Jafarey, A. (2010). Gathas: los cantos divinos de Zaratustra. Createspace.

Alter, K.; Dawans, V. (2006). The integrated approach to social entrepreneurship: building high performance organizations. SER (Social enterprise reporter), (204), 1-4. Recuperado de https://bit.ly/2ZWYrcN

Ander-Egg, E. (2003). Repensando la investigación acción participativa. Buenos Aires: Lumen Humanitas.

Balamatsias, D. (04 de mayo de 2018). Social Innovation. 8 popular social innovation definitions. Social Innovation Academy. Recuperado de https://bit.ly/2XQWRXd

Barab, S.; Thomas, M.; Dodge, T.; Goodrich, T.; Carteaux, B.; Tuzun, H. (2002). Empowerment design work: building participant structures that transform. En P. Bell, R. Stevens; T. Satwicz (Eds.) Keeping learning complex: The Proceedings of the Fifth International Conference of the Learning Sciences (ICLS). (132-138). Mahwah, NJ: Lawrence Erlbaum Associates.

Board of Innovation (s. f.). Business model kit. Recuperado de https://bit.ly/3cq8w4B

Buckland, H.; Murillo, D. (2014). La innovación social en América Latina. Marco conceptual y agentes. Instituto de Innovación Socia, Esade-Fomin. Recuperado de https://bit.ly/3gImrq6

Brown, T.: Wyatt, J. (2010). Design thinking for social innovation. Stanford Social Innovation Review. DOI: https://doi. org/10.1596/1020-797X_12_1_29 resultan en nuevas expectativas y necesidades que no fueron experimentadas antes por los miembros de estas comunidades. Esto en razón a que la producción, más allá de las necesidades de sustento y del comercio, no son actividades que están inmersas en la cultura indígena. Así, entonces, un proceso de acompañamiento debe ir desde la identificación de vocaciones productivas hasta la consolidación de productos, distribución y procesos de venta.

Dado que las iniciativas empresariales se basan en actividades que forman parte de la vida cotidiana de la comunidad, es de vital importancia que los individuos comprendan el alcance del proyecto, lo vivan como suyo, logren la motivación necesaria para su puesta en marcha, se apropien, se empoderen de él y busquen mecanismos de construcción y mejoramiento.

DANE (Departamento Administrativo Nacional de Estadística). (2005). Censo General 2005. Bogotá: DANE. Recuperado de https://bit.ly/36NYclO

Design Council. (2007). A study of the design process. Eleven lessons: managing design in eleven global brands. Recuperado de https://bit.ly/371AtyN

Hernández, J.; Tirado, P.; Ariza, A. (2016). El concepto de innovación social: ámbitos, definiciones y alcances teóricos. CIRIEC-España, Revista de Economía Pública, Social y Cooperativa, (88), 165-199. DOI: https://doi.org/10.7203/ CIRIEC-E.88.8849

IDEO. (2015). The field guide to human-centered design. Recuperado de https://bit.ly/3eCrsyw

Inglesis-Barcellos, E. E.; Botura, G. (2018). Design thinking: usercentered multidisciplinary methodology based on people and innovation. En J. Kantola; T. Barath; S. Nazir (Eds.) Advances in human factors, business management and leadership. AHFE 2017. Advances in Intelligent Systems and Computing (354366). Cham: Springer. DOI: https://doi.org/10.1007/978-3319-60372-8_17

Jiménez, E. (2017). Análisis y desarrollo de un modelo de tutorización integral basado en el design thinking orientado a la innovación estratégica en empresas colombianas (Tesis doctoral). Universidad Politécnica de Valencia, España. Recuperado de https://riunet.upv.es/handle/10251/90557

Maccallum, D.; Moulaert, F.; Hillier, J.; Vicari, S. (Eds.). (2009). Social innovation and territorial development. Farnham: Ashgate. 
Mulgan, G.; Tucker, S.; Ali, R.; Sanders, B. (2007). Social innovation: what it is, why it matters and how it can be accelerated. Londres: The Young Foundation.

Murray, R.; Caulier-Grice, J.; Mulgan, G. (2010). The open book of social innovation. Gran Bretaña: Nesta.

OCDE (Organización para la Cooperación y el Desarrollo Económicos); Eurostat. (2006). Manual de Oslo. Madrid: Grupo Tragsa. Recuperado de https://bit.ly/3eHNUWS

ONIC (Organización Nacional Indígena de Colombia). (25 de enero de 2018). La ONIC ratifica posible exterminio estadístico en el Censo 2018 por incumplimiento de acuerdos. Recuperado de https://bit.ly/2BkXbWB

Parada, J.; Ganga, F.; y Rivera, Y. (2017). Estado del arte de la innovación social: una mirada a la perspectiva de Europa y Latinoamérica. Opción, 33(82), 563-587. Recuperado de https://bit.ly/3ciGYy0

Patiño,O.; Ruiz, C.; Echeverry, P. (2018). Los proyectos de emprendimiento social en comunidades indígenas. Libro: la gerencia de proyectos como impulsor de la estrategia organizacional. Bogotá: Ediciones EAN.

Phills, J.; Deiglmeier, K.; Miller, D. (2008). Rediscovering social innovation. Stanford Social Innovation Review. Recuperado de https://ssir.org/ articles/entry/rediscovering_social_innovation
PNUD (Programa de las Naciones Unidas para el Desarrollo). (2015). Objetivos de desarrollo sostenible. Recuperado de https://bit.ly/2XOVfxl

Richard, D. (2013). How to start a creative business: the jargonfree guide for creative entrepreneurs. Newton Abbot: David and Charles.

Sanders, E.; Stappers, P.J. (2012). Convivial toolbox. Generative research for the front end of design. Amsterdam: BIS Publishers.

Social Enterprise Alliance. (2019). What is Social Enterprise? Recuperado de https://bit.ly/2Ax0sl7

The Young Foundation (2012). Social Innovation Overview: A deliverable of the project: The theoretical, empirical and policy foundations for building social innovation in Europe (TEPSIE), Brussels: European Commission, DG Research. Recuperado de https://bit.ly/3drCt54

Westley, F. R.; McConnell, J. W. (2010). Making a difference. Strategies for scaling social innovation for greater impact. The Innovation Journal: The Public Sector Innovation Journal, 15(2), 2-19. Recuperado de https://bit.ly/3gGzUyB

Zimmerman, M. A. (2000) Empowerment theory. En J. Rappaport, E. Seidman (Eds.) Handbook of Community Psychology. (43-63). Boston, MA: Springer. DOI: https://doi. org/10.1007/978-1-4615-4193-6_2 